\title{
What can we learn from gene expression profiling of mouse oocytes?
}

\author{
Toshio Hamatani ${ }^{1,2}$, Mitsutoshi Yamada ${ }^{1,2}$, Hidenori Akutsu ${ }^{2}$, Naoaki Kuji ${ }^{1}$, \\ Yoshiyuki Mochimaru ${ }^{1}$, Mitsuko Takano ${ }^{1}$, Masashi Toyoda $^{2}$, Kenji Miyado ${ }^{2}$, Akihiro Umezawa ${ }^{2}$ \\ and Yasunori Yoshimura ${ }^{1}$ \\ ${ }^{1}$ Department of Obstetrics and Gynecology, Keio University School of Medicine, 35 Shinanomachi Shijuku-ku, Tokyo \\ 160-8582, Japan and ${ }^{2}$ Department of Reproductive Biology and Pathology, National Institute for Child Health and \\ Development, 2-10-1 Okura Setagaya-ku, Tokyo 157-8535, Japan
}

Correspondence should be addressed to T Hamatani; Email: t-hama@sc.itc.keio.ac.jp

\begin{abstract}
Mammalian ooplasm supports the preimplantation development and reprograms the introduced nucleus transferred from a somatic cell to confer pluripotency in a cloning experiment. However, the underlying molecular mechanisms of oocyte competence remain unknown. Recent advances in microarray technologies have allowed gene expression profiling of such tiny specimens as oocytes and preimplantation embryos, generating a flood of information about gene expressions. So, what can we learn from it? Here, we review the initiative global gene expression studies of mouse and/or human oocytes, focusing on the lists of maternal transcripts and their expression patterns during oogenesis and preimplantation development. Especially, the genes expressed exclusively in oocytes should contribute to the uniqueness of oocyte competence, driving mammalian development systems of oocytes and preimplantation embryos. Furthermore, we discuss future directions for oocyte gene expression profiling, including discovering biomarkers of oocyte quality and exploiting the microarray data for 'making oocytes'.

Reproduction (2008) 135 581-592
\end{abstract}

\section{Introduction}

The mammalian oocyte is marked by extraordinary biological competence; it can haploidize its DNA, be fertilized and reprogram the sperm chromatin into a functional pronucleus, induce zygotic genome activation (ZGA), give rise to totipotency, and drive early embryonic development. Using its ability to reprogram a somatic nucleus transferred into an enucleated oocyte, derivation of embryonic stem (ES) cells from cloned blastocysts for therapeutic cloning is explored. The molecular mechanisms underlining such oocyte competence are largely unknown.

On the other hand, the reproductive capacity of women declines dramatically beyond the mid-30s (van Kooij et al. 1996, ASRM/SART 2000, Armstrong 2001, Klein \& Sauer 2001), which is mainly caused by agerelated decline in oocyte quality. For example, young women undergoing standard in vitro fertilization (IVF) with their own eggs show a success rate comparable with older women ( $>40$ years) undergoing IVF with eggs donated by this younger subset of women (Navot et al. 1991). To overcome age-related decline in oocyte quality, ooplasmic donation has been performed by injecting ooplasm from a young, healthy donor oocyte into a patient oocyte to improve the outcome of assisted reproduction techniques (Cohen et al. 1997, 1998, Takeuchi et al. 1999). There is, however, little molecular evidence supporting the efficacy and the safety of ooplasmic donation. Furthermore, no molecular biomarker for oocyte quality has been established. Oocyte quality based on a morphological grading system is the only reliable prognostic factor in human IVF programs. Studies of molecular mechanisms involved in oocyte quality could have important implications for the efficacy and safety of clinical ooplasmic donation.

Thus, understanding the molecular mechanisms in oocytes is quite important for both reproductive biology and regenerative medicine. The scarcity of the materials, however, both in size (diameter $<100 \mathrm{um)}$ and in quantity (only a few to tens of oocytes from each ovulation in mice), has hampered the molecular analysis of oocytes. Earlier attempts to analyze oocytes employed RT-PCR and differential display using only a few candidate genes. In addition, serial analysis of gene expression (SAGE) and cDNA libraries were generated from mouse and human oocytes, and SAGE tags and expressed sequence tags (ESTs) were sequenced for rapid gene discovery and expression profiling in oocytes 
(Ko et al. 2000, Ko 2004, Adjaye 2005, Evsikov et al. 2006). Furthermore, the recent progress in RNA amplification methods and microarray platforms including genes unique to oocytes and preimplantation embryos allows us to apply global gene expression profiling to the studies of the oocytes and preimplantation embryos (Carter et al. 2003). To date, several reports of the oocyte transcriptome using unique biological models have been published (Dobson et al. 2004, Hamatani et al. 2004a, 2004b, Wang et al. 2004, Zeng et al. 2004, Pan et al. 2005, Assou et al. 2006, Kocabas et al. 2006, Yoon et al. 2006). The identification of a large number of genes expressed in oocytes, especially oocyte-specific genes, and multiple signaling pathways in the models by such global gene expression profiling is the first step toward understanding oocyte quality and the molecular mechanisms underlying oogenesis, developmental programs, and totipotency in preimplantation embryos.

\section{Global gene expression profiling of mouse preimplantation embryos to dissect maternal transcripts}

Two groups simultaneously published the first reports on global gene expression profiling of all stages of preimplantation embryos (Fig. 1; Hamatani et al. 2004a, Wang et al. 2004). While Wang et al. used the Affymetrix 25-mer oligo DNA microarray system, we used the NIA 60-mer oligo microarray (Agilent Mouse Development Array), which is enriched for genes expressed in stem cells and preimplantation embryos (Carter et al. 2003). Taking advantage of 60-mer oligo DNA hybridization kinetics (Hughes et al. 2001), it was also optimized for use with tiny amounts of RNA (Carter et al. 2003). During preimplantation development, 12179 out of 21939 gene features on the NIA 60-mer oligo microarray showed statistically significant changes with false discovery rate (FDR) $<10 \%$ by ANOVA-FDR test. Pair-wise comparison, hierarchical clustering analysis, and principal component analysis (PCA) revealed two major transient waves of de novo transcription (Fig. 1A-C). The first wave corresponds to ZGA. The second wave, mid-preimplantation gene activation (MGA), contributes to dramatic morphological changes during late preimplantation development.

To trace the expression changes of individual genes, 12179 statistically significant genes were analyzed by $k$-means clustering method and 9 clusters were identified (Fig. 2). Gene expression patterns of these clusters can be assigned to three main groups. The first group appears to represent ZGA genes that are first activated from the zygotic genome (Clusters 1, 4, 5, and 8). The list of the ZGA genes suggests that ZGA is not promiscuous as previously proposed and contributes mainly to the preparation of basic cellular machinery during the twocell and the four-cell stages.
The second group represents maternal transcripts with distinctive patterns of degradation during preimplantation development (Clusters 7 and 9). Although the massive maternal RNA degradation pattern by the twocell stage is confirmed (Cluster 9) as previous studies suggested (Nothias et al. 1995, Schultz 2002), 70.5 and $32.5 \%$ of the transcripts in Clusters 7 and 9 respectively further show significant reduction from the four-cell to eight-cell stages. Selective degradation of maternal transcripts during oocyte maturation is, as also shown by the latest study (Su et al. 2007), a developmentally regulated event preceding the transition of gene expression from maternal to zygotic control. Since most genes in Clusters 7 and 9 are not reactivated during preimplantation development, the genes in these clusters are suggested to have specific functions either in oogenesis, oocyte maturation, fertilization, and/or early phases of preimplantation development.

The third group appears to represent genes that follow a combination of these two patterns (Clusters 2 and 3); 3329 genes whose expression first significantly increase from the four-cell to eight-cell stages are identified as the MGA genes, and 82.7 and $12.3 \%$ of them fall into Clusters 2 and 3 respectively. Further expression profiling of embryos treated with inhibitors of transcription and translation reveals that the translation of maternal RNAs is required for the initiation of ZGA, suggesting a cascade of gene activation from maternal $\mathrm{RNA} /$ protein sets to ZGA gene sets and thence to MGA gene sets (Hamatani et al. 2004a).

By MAPPFinder (Dahlquist et al. 2002, Doniger et al. 2003), which is a tool to identify global biological trends in gene expression data by interacting the annotations of Gene Ontology (GO) terms (Ashburner et al. 2000), the genes in the clusters of maternal transcripts are associated to such GO terms as 'circadian rhythm,' 'M-phase of mitotic cell cycle,' 'DNA replication,' 'Golgi apparatus/ intracellular protein transport,' 'adherent junction,' 'small GTPase regulatory/interacting protein,' and 'intracellular signaling cascade'. The 'circadian rhythm' category includes seven mammal known circadian genes: Per1-3, Cry1-2, Bmal1/Arntl, and Clock. The transcripts of Bmal1/Arntl, Clock, Timeless, Cry1, and Csnk1e decrease during the one-cell to two-cell stages as previous reports showed (Johnson et al. 2002).

The egg-sperm fusion at fertilization in mammals releases an oocyte from metaphase II arrest by increasing $\mathrm{Ca}^{2+}$ levels, activating $\mathrm{Ca}^{2+}$-calmodulin kinase $\mathrm{II}$, and targeting cyclin B and $\mathrm{c}-\mathrm{mos}$ for degradation via the ubiquitin-proteasome pathway. Rfp/4, an E3 ubiquitin protein ligase, regulates the degradation of cyclin B1 (Ccnb1) protein (Cluster 6b) (Suzumori et al. 2003), which is a well-known example of a transcript with a short $\operatorname{poly}(\mathrm{A})$ tail that is regulated at the post-transcriptional level in oocytes. Furthermore, Cpeb, Eif4e, Cpsf2, and Stk13/Aurkc, which are involved in the masking and/or translational regulation of transcripts with short 
A

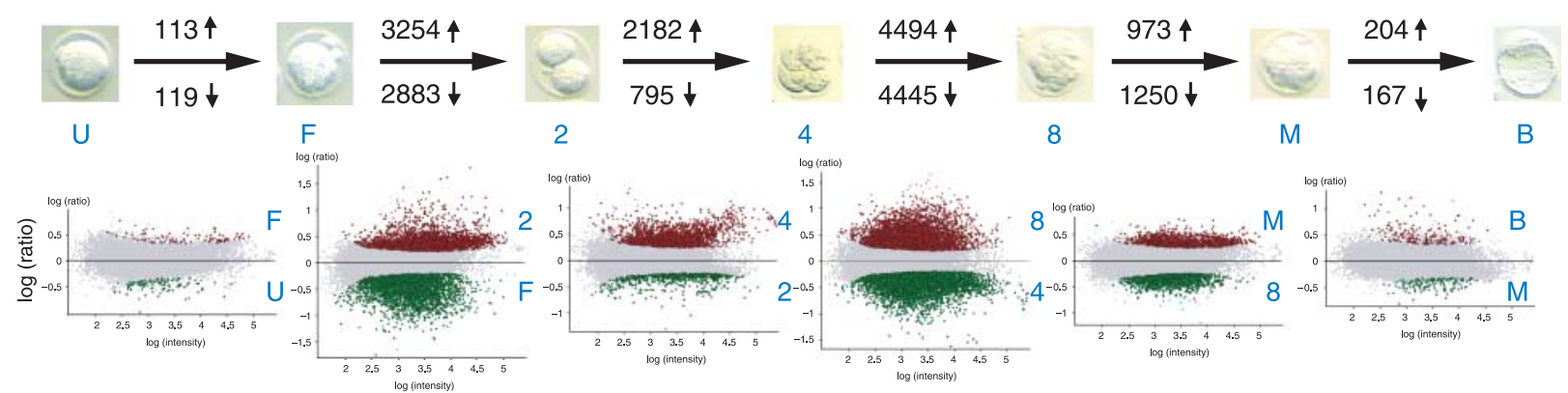

B

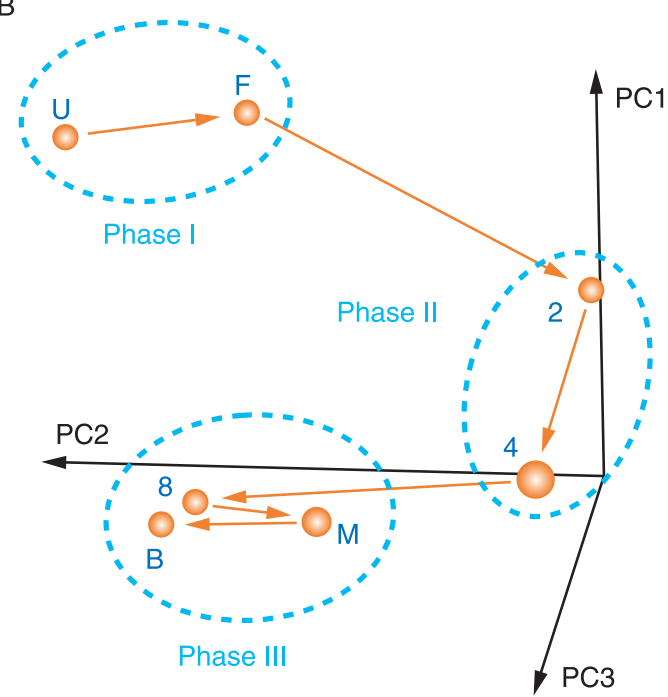

C

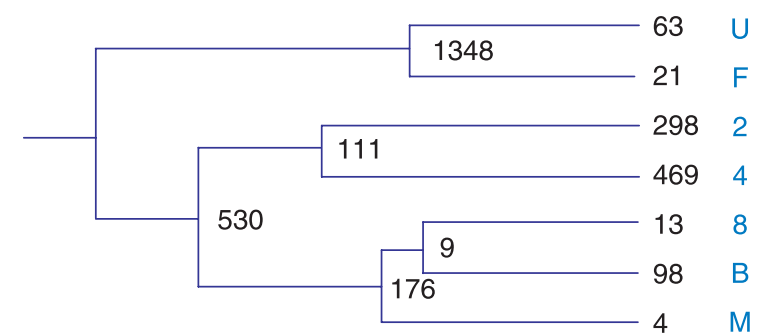

1.0

0.8

0.20

$0.6 \quad 0.4$

Distance

Figure 1 Global outlook of gene expression during preimplantation development (reprinted from 'Dynamics of global gene expression changes during mouse preimplantation development', Hamatani T et al. 2004 Developmental Cell 6 117-131, with permission from Elsevier). (A) A matrix of scatter plots. U, F, 2, 4, 8, M, and B denote unfertilized egg, fertilized egg, two-cell embryo, four-cell embryo, eight-cell embryo, morula, and blastocyst respectively. Each scatter plot shows the comparison of gene expression between embryo stages. A horizontal axis represents the averaged $\log$ (intensity) of genes, whereas a vertical axis represents the log (ratio) of signal intensity for each gene between one stage and another stage. Colored spots (red and green) represent genes that passed the $\mathrm{FDR}=10 \%$ statistical test. Red spots represent array features with higher expression at a later stage, whereas green spots represent array features with lower expression. (B) Principal component analysis. (C) Hierarchical clustering analysis. Numerical values represent the number of genes specific to each cluster or stage. A list of these stage-specific genes are available at the web site of Cell Press (Hamatani et al. 2004a).

poly(A) tails in oocytes (Hodgman et al. 2001, Mendez \& Richter 2001), also decrease their transcripts by the twocell stage. The presence of the 'DNA replication' category in oocytes indicates that oocytes are already well equipped with DNA replication machinery, as exemplified by the fact that neither the lack of Zar1 (Wu et al. 2003) nor the presence of jasplakinolide, which is the most powerful known microfilament inhibitor (Terada et al. 2000), can prevent the initiation of DNA replication. In another global gene expression study of preimplantation embryos, DNA repair genes are also over-represented at the oocyte stage when compared with the one-cell through the blastocyst stages in their transcript profiling during preimplantation development (Zeng et al. 2004). Genes that are downregulated from oocytes to two-cell embryos include many genes involved in DNA repairs, including Orc1l, Orc4l, Orc5l, Orc6l, Mcm4, Pcna, Pola2, Polm, Blm,
Top1, and Msh6 (Cluster 9); Msh3 and Mcm7 (Cluster 7); and $C d c 7 / 1 / C d c 7, C d c 45 I, C c n a 2$, and Dbf4/Ask (Cluster 6). Furthermore, another group searched for maternal transcripts of polarity-regulating genes in mouse oocytes by global gene expression profiling of preimplantation embryos, which may subsequently control polarity in preimplantation embryos (Wang et al. 2004). They focused on three genes whose homologs have been shown to regulate cellular polarity in Drosophila: Flamingo, dystroglycan1 (Dag1), and cornichon (Cnih2) both of which are included by Cluster 3 .

\section{Global gene expression changes during oogenesis}

Although several groups have studied global gene expression in human and mouse oocytes at the later stages of folliculogenesis (germinal vesicle stage and metaphase II stage; Wang et al. 2004, Cui et al. 2007, 
A
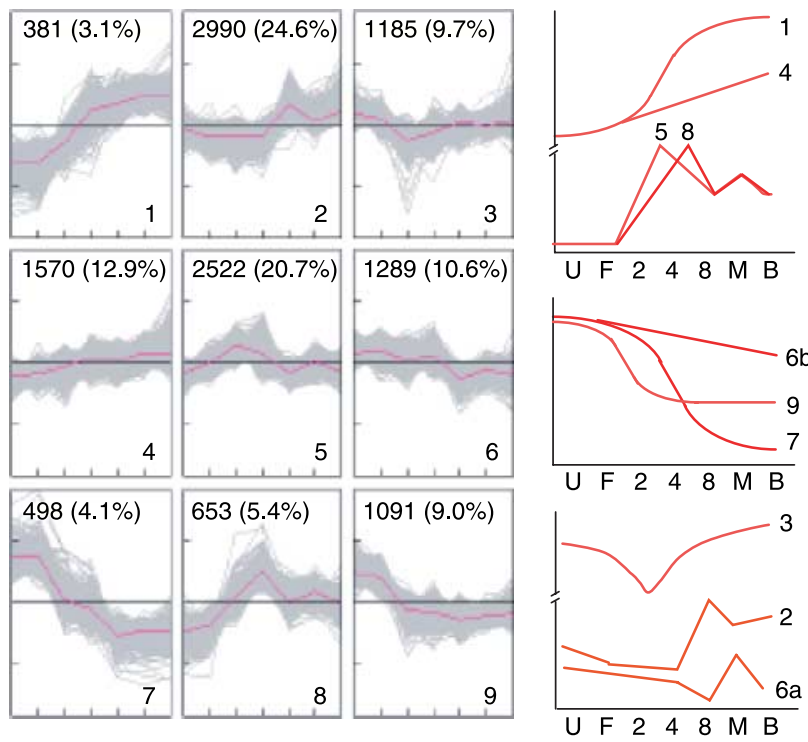

$\mathrm{B}$

\begin{tabular}{|c|c|c|}
\hline Cluster 6 & $\frac{\text { Cluster } 7}{G d f 9}$ & $\frac{\text { Cluster } 9}{M o s / c-m o s}$ \\
\hline $\begin{array}{c}\text { Mater } \\
\text { Ddx4/Vasa }\end{array}$ & $\begin{array}{c}\text { Gdf9 } \\
\text { Obox1 }\end{array}$ & $\begin{array}{c}\text { Mos/c-mos } \\
\text { Kit/c-kit }\end{array}$ \\
\hline $\begin{array}{c}\text { Dax4lvasa } \\
\text { Zp3 }\end{array}$ & Ezh2 & Stat3 \\
\hline Gata4 & Mad2/2 & Cry 1 \\
\hline Frap $1 / m T O R$ & Tcl1 & Oosp1 \\
\hline Fsrg1/Brd2 & $\begin{array}{l}\text { Bmit } \\
\text { Bmoltiantt }\end{array}$ & Nobox/Og2x \\
\hline Baz1a & $\begin{array}{c}\text { Bmal1/Arnt } \\
\text { Clock }\end{array}$ & $\begin{array}{l}\text { Cpeb1 } \\
\text { Magoh }\end{array}$ \\
\hline Cdc45I & Zar1 & Catna1 \\
\hline$D t r / H B-E G F$ & Rfpl4 & $N$-cadherin \\
\hline Smarca5 & $\begin{array}{c}\text { Eps8 } \\
\text { Ep }\end{array}$ & Hells/Lysh \\
\hline Brd4 & Stk13/AurkC & Morf4/2/MrgX \\
\hline Son & Zfp68 & Baz1b \\
\hline Nfkbia/lkBa & $R n f 35 / 2 c z 61$ & $Z p 1$ \\
\hline$N f k b 1 / N F-k B$ & $\begin{array}{l}\text { Sfmbt1 } \\
\text { Spindlin }\end{array}$ & $\begin{array}{c}Z p 2 \\
\text { Scm/2 }\end{array}$ \\
\hline
\end{tabular}

Figure 2 Time-course analysis of individual genes. (Reprinted from 'Dynamics of global gene expression changes during mouse preimplantation development', Hamatani T et al. 2004 Developmental Cell 6 117-131, with permission from Elsevier). (A) General trends of expression changes analyzed by $k$-means clustering method. The nine clusters were further classified into three super groups by visual inspection as shown in the three schemes in the far right column. (B) Representative genes in each cluster.

Gasca et al. 2007, Zhang et al. 2007), only two groups successfully performed global gene expression studies using mouse oocytes at the very early stages of folliculogenesis (Pan et al. 2005, Yoon et al. 2006). Pan et al. (2005) compared the transcriptomes of mouse oocytes obtained from day 2 primordial follicles to day 22 equine CG primed large antral follicles. From the primordial to large antral stages, 18529 probe sets corresponding to 11766 unigenes detected significant gene expression in oocytes that developed in vivo. The hierarchical clustering dendrogram and PCA analysis showed that the primordial oocyte is separated from oocytes obtained from the other stages. Many important genes encoding 'secreted proteins', which are defined on their own terms in that manuscript, display marked upregulation between the primordial and primary follicle stages (e.g., Gdf9, Bmp15, Bmp5, Bmp6, Tgfb2, $T g f b 3$, and several genes related to Notch, Shh, and Egf signaling pathways). Thus, the primordial to primary follicle transition is a major transition and likely reflects the dramatic reorganization in follicle structure and initiation of growth and development. Of the 16883 probe sets differentially detected between these stages, 5020 display a twofold change in relative abundance. Another apparent transition occurs between oocytes obtained from secondary follicles and those from small antral follicles, which corresponds to the acquisition of meiotic competence. The 736 probe sets of which $\sim 65 \%$ are downregulated display a significant twofold change at this transition.

The principal component-based clustering shows three distinct patterns of gene expression. The first pattern shows consistent increase or decrease throughout the oocyte development and the most dramatic changes from the primordial to primary follicle stages, which the bulk of genes (10 117 probe sets) display (Fig. 3A). The second pattern peaks or hits the bottom at the primary follicle stage (Fig. 3B) and the third one shows the dynamic expression changes from the primary to the secondary follicle stages (Fig. 3C). The Expression Analysis Systematic Explorer software (http://david.abcc. ncifcrf.gov/ease/ease.jsp) for discovery of biological themes within the list of genes also shows the overrepresentation of genes involved in DNA repair and response to DNA damage throughout oocyte development, suggesting a protective mechanism to insure genomic integrity of the female germ line.

In addition, by analysis of global gene expression profiling of oocytes during the germinal vesicle stage to the metaphase II stage, new potential regulators and marker genes for oocyte maturation have been identified: Pacsin2, Map2k (Cui et al. 2007), and the genes related to BRCA1 regulation pathway, including Bard1, Rbbp4, Brap, Rbbp7, Rbl2, Bub3, and Bub1b (Gasca et al. 2007).

\section{Global gene expression changes during loss of oocyte quality}

To elucidate factors determining oocyte quality, a mouse model highlighting the age-related decline in fertility and oocyte quality was used (Hamatani et al. 2004b, Steuerwald et al. 2007). The expression profiles of metaphase II oocytes collected from 5- to 6-week-old mice were compared with those collected from 42- to 45-week-old mice using the NIA 60-mer oligo microarray (Hamatani et al. 2004b). Among 11000 genes whose transcripts were detected in oocytes, about 5\% (530) showed statistically significant expression changes, 

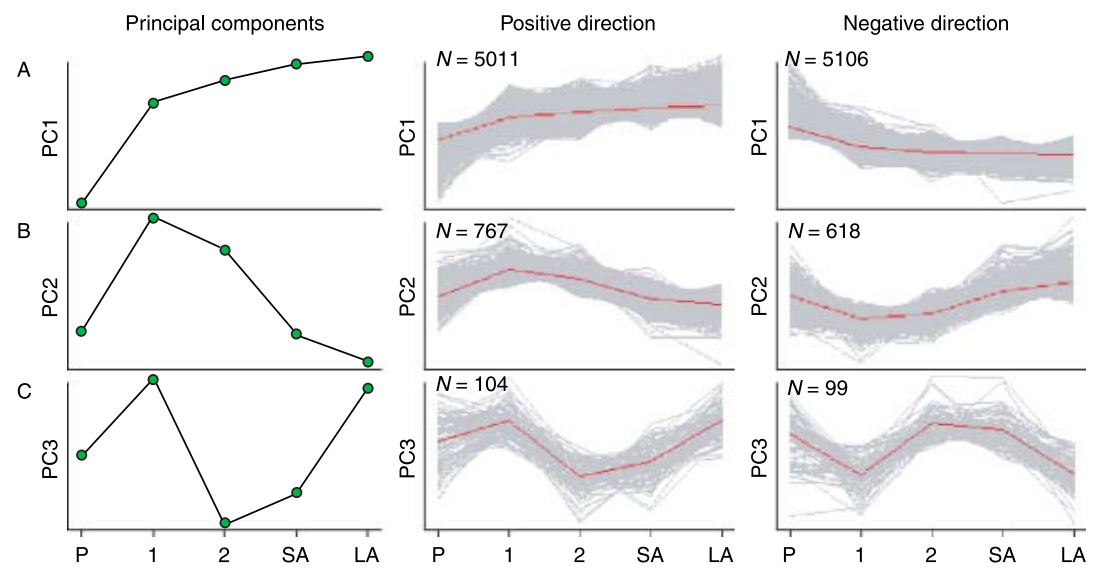

Figure 3 Principal component-based clustering to analyze gene expression profiles of oocytes during the primordial follicle stage to the large antral follicle stage (Pan et al. 2005) by NIA array analysis tool (http://lgsun.grc.nia.nih.gov/ANOVA/). The NIA Array Analysis tool identifies two clusters associated with a given pattern: genes positively and negatively correlated with the pattern. P, 1, 2, $\mathrm{SA}$, and LA represent the primordial follicle stage, the primary follicle stage, the secondary follicle stage, the small antral follicle stage, and the large antral follicle stage respectively.

excluding the possibility of global decline in transcript abundance. Consistent with the generally accepted view of aging, the differentially expressed genes include ones involved in mitochondrial function and oxidative stress. Interestingly, a new non-invasive and highly sensitive method for measuring cellular respiration with scanning electrochemical microscopy shows that decreased cellular respiration in oocytes from aged mice is associated with impaired preimplantation development (Abe 2007). However, the expression of other genes involved in chromatin structure, DNA methylation, genome stability, and RNA helicases are also altered, suggesting the existence of additional mechanisms for aging in oocytes. For example, the decreased Dnmt1 (Dnmt1o and Dnmt1s) expression and the increased Dnmt3b during aging are observed in oocytes. Because the same pattern of expression change in Dnmt genes has already been reported in aging WI-38 fibroblast cells (Lopatina et al. 2002), the genomic methylation patterns are suggested to be altered in aging cells. Telomerase reverse transcriptase and yeast mutant $\mathrm{H} / \mathrm{L} / \mathrm{S}$ mismatch repair gene homologs are also downregulated during aging. Interestingly, more than 30 zinc finger proteins are shown as the downregulated genes during aging. Furthermore, we identified and characterized a group of new oocyte-specific mouse genes, members of the human NACHT, leucine rich repeat and pyrin domain containing (NALP/NLRP) gene family among the transcripts decreased with aging. The Nalp gene family includes Mater/Nalp5/N/rp5 whose null mutant embryos arrest cleavage at the two-cell stage (Tong et al. 2000), suggesting an important role of this gene family in oogenesis, fertilization, and/or preimplantation development. These results have implications for aging research as well as for clinical ooplasmic donation to rejuvenate aging oocytes.

Polycystic ovary syndrome (PCOS) is another good model for studying loss of oocyte quality. The reproductive performance of women undergoing IVF treatment with PCOS is characterized by their good response to ovarian stimulation that yields higher number of oocytes; however, with lower implantation and higher miscarriage rates (Engmann et al. 1999, Ludwig et al. 1999, Mulders et al. 2003). Individual oocytes retrieved from nine women with PCOS and that from ten non-hirsute ovulatory women are used for microarray hybridization (Wood et al. 2007). Of the 8123 transcripts expressed in metaphase II oocytes, 374 show significant differences in mRNA abundance in the PCOS oocyte. The genes associated with chromosome alignment and centrosome, and the genes containing putative androgen receptors and/or PPAR $\gamma$-binding sites are upregulated. The expression of these genes, which is generally not a part of the human oocyte transcriptome, is suggested to contribute to abnormalities in early embryonic development. Furthermore, upregulation of maternaleffect genes are notable. Although only seven mammalian maternal-effect genes (Mater/NIrp5, Hsf1, Dnmt1, Zar1, Npm2, Stella, Fmn2, and Bnc1) have been identified to date, three (Mater/NIrp5, Fmn2, and Bnc1) are upregulated. Increased expression of maternal-effect genes may negatively impact embryonic development.

Dielectrophoresis is a potential non-invasive method to select oocytes of good quality. In fact, dielectrophoretically separated in vitro-derived bovine metaphase II oocytes show a difference in the rate of blastocyst development and significant difference in transcriptional abundance of 36 genes as a result from global gene expression profiling. This suggests that dielectrophoretic behavior and the 36 genes including Anxa2, Ptgs2, and Dnmt1 are potential biomarkers for oocyte quality (Dessie et al. 2007).

Recently, microarray technology was also applied to screening for chromosomal anomalies: comparative genomic hybridization $(\mathrm{CGH})$ is used to assess the copy number of chromosomes in polar bodies and oocytes (Wells et al. 2002, Fragouli et al. 2006). CGH has the major advantage that every chromosome is tested, rather than the limited subset assessed using fluorescence in situ hybridization (FISH). The CGH protocols, which allow efficient DNA amplification from single cells and 
reduce the amount of time required for the analysis, are currently undergoing preclinical testing in a number of preimplantation genetic diagnosis laboratories (Patrizio et al. 2007).

\section{Identification of oocyte-specific transcripts and their clustering in the mouse genome}

A mammalian oocyte is the only known cell that can activate a zygotic genome after fertilization and reprogram a somatic nucleus transferred from a differentiated cell in cloning experiments. Therefore, several genes specifically expressed in oocytes are likely responsible for the ability to reprogram genomes as well as for oogenesis. It is the case for the so-called maternal genes such as Mater, Zar1, and Npm2 that are all required for normal embryonic development beyond the one-cell or two-cell stage (Fig. 4A; Tong et al. 2000, Dean 2002, Burns et al. 2003, Wu et al. 2003). Gdf9 and Bmp15 are also known to play important roles in female germ cells during folliculogenesis (Dong et al. 1996, Galloway et al. 2000). Accordingly, genes specifically expressed in the oocyte seem to control oogenesis, ovarian folliculogenesis, and preimplantation development.

In attempts to identify novel oocyte-specific genes, several groups have used mRNA differential display (Zeng \& Schultz 2003), suppression subtractive hybridization (Hennebold et al. 2000), and in silico subtraction approaches (Rajkovic et al. 2001, Dade et al. 2003). It is, however, essential to analyze all transcripts/genes in a wide selection of organs and cell types including totipotent fertilized eggs, pluripotent embryonic cells, a variety of adult stem cells, and terminally differentiated cells. Sharov et al. (2003) obtained 249200 high-quality EST sequences from the NCBI Unigene database that included a broad collection of NIA mouse cDNA libraries and clustered them into $\sim 30000$ gene indexes including 977 previously unidentified genes. By analyzing the expression levels of the gene indexes based on the frequencies of the corresponding ESTs in Unigene cDNA libraries, genes that characterize oocytes and preimplantation embryos are identified (Sharov et al. 2003). Furthermore, the gene expression specificity to oocytes or/and preimplantation embryos is validated using gene expression profiling data of female germ cells during oogenesis and preimplantation embryos (Hamatani et al. 2004a, Wang et al. 2004, Pan et al. 2005). Several example of genes preferentially expressed in oocytes are selected and their gene expression levels are demonstrated to increase in oocytes during oogenesis (from the primordial follicle stage to the large antral follicle stage) and decrease during preimplantation development (from unfertilized egg to blastocyst) by the microarray experiments (Fig. 4B). Mager et al. (2006) also identified 51 genes as candidate maternal-effect genes in silico (always not present during the two-cell through the eight-cell or at the blastocyst stage), by comparing published results of three independent studies of mouse preimplantation embryo transcriptomes (Hamatani et al. 2004a, Wang et al. 2004, Zeng et al. 2004).

The group that found six genes of the mouse oogenesin family reported that not a few loci near the telomere in the mouse genome contain several genes specifically expressed in oocytes (Paillisson et al. 2005). Mouse oogenesin family genes are expressed exclusively in oocytes and present on chromosome 4 in a cluster of almost $1 \mathrm{Mb}$ composed of 12 oogenesin paralogous genes. On the other hand, we also identified nine novel genes presenting similarities with Mater/Nalp5 (Tong et al. 2000) and expression specific to oocytes, seven of which are clusterized on a certain locus of chromosome 7 (Fig. 5). The gene expression specificity of the novel Nalp-family genes to oocytes has been experimentally validated using Northern blot and in situ hybridization (Hamatani et al. 2004b; Fig. 6). Recently, we further identified a group of oocyte-specific genes encoding zinc finger proteins that clusterize in a near-telomere locus of chromosomes 6 and 11 (unpublished data). Telomeric regions of chromosomes are mainly composed of heterochromatin in most eukaryotic genomes. Because gene silencing near the telomere has been known and called 'telomere position effect' in Drosophila and yeast, the specific near-telomere position of the clusters of oocyte-specific genes in mice may contribute toward their gene silencing in non-ovarian tissues.

There is another noted attempt to identify important genes that are preferentially expressed in oocytes and conserved in chordates. Evsikov et al. (2006) compared the collection of ESTs from their mouse oocyte libraries to those from the eggs of Xenopus and ascidians to extract conserved genes that are expressed in chordate oocytes. More than $50 \%$ of the genes expressed in mouse oocyte libraries are also expressed in the eggs of Xenopus and ascidians. To investigate the evolutionary hardwired molecular pathways shared among chordates, GO term frequencies in 2090 genes that are commonly expressed in eggs of all three species are compared with those in the entire set of genes expressed in their mouse oocyte library. Although this analysis shows a substantial overlap with GO terms (biological process) associated with housekeeping genes, several GO terms (molecular function) such as 'motor activity,' 'small protein activating enzyme activity,' 'transferase activity,' 'helicase activity,' and two specific signal transducer activities (serine/threonine kinase activity and ligand-dependent nuclear receptor activity) are over-represented and provide a snapshot of gene functions shared particularly by the chordate oocytes. Another study group used a multi-species cDNA microarray containing 3456 transcripts from three distinct cDNA libraries from bovine, mouse, and Xenopus oocytes (Vallee et al. 2006). The cross-species hybridizations reveal that 1541 positive hybridization signals are generated by oocytes of all three species, and 268 of these, including SMFN (small fragment nuclease), Spin (spindlin), and 
A
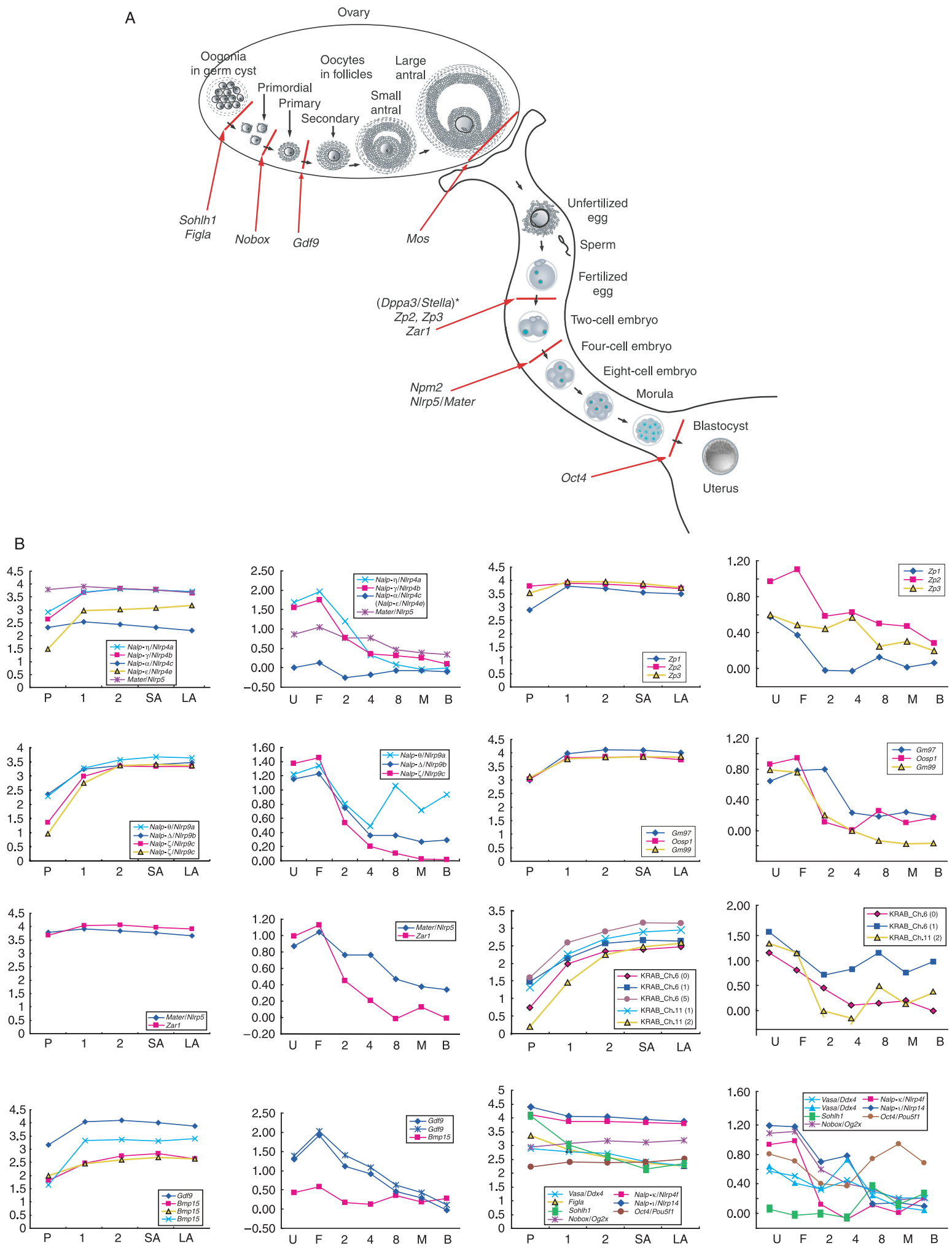

Figure 4 (A) Knockout mouse phenotypes of genes preferentially expressed in oocytes. *Development of embryos from Stella-/ - intercrosses starts to be affected from $1.5 \mathrm{dpc}$ onward (the two-cell stage) and only a low percentage reach the blastocyst stage by $3.5 \mathrm{dpc}$. (B) The gene expression changes of several genes known as oocyte specific. The oocyte-specific genes, including Nalps, showed increased expression during oogenesis and decreased expression during preimplantation development in the global gene expression studies. P, 1, 2, SA, and LA represent the primordial follicle stage, the primary follicle stage, the secondary follicle stage, the small antral follicle stage, and the large antral follicle stage respectively. $U$, F, 2, 4, 8, $\mathrm{M}$, and B denote unfertilized egg, fertilized egg, two-cell embryo, four-cell embryo, eight-cell embryo, morula, and blastocyst respectively. 


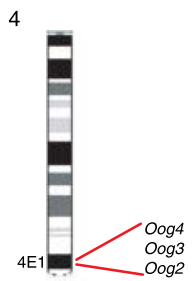

9

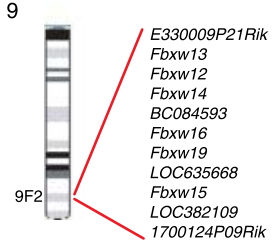

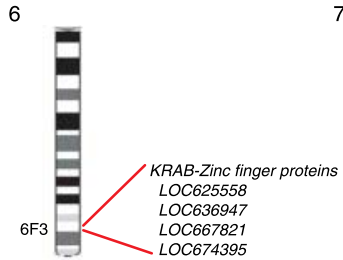

12

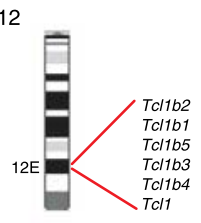

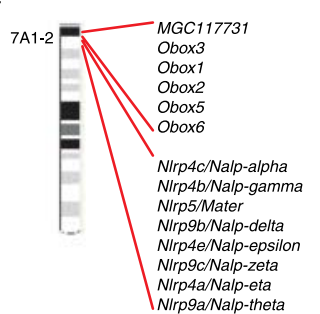

19

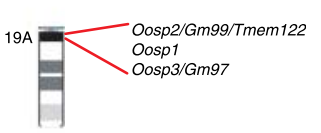

Figure 5 Clusters of oocyte-specific genes on mouse chromosomes 4, 6, 7, 9, 12, and 19 .

PRMT1 (protein arginine methyltransferase 1) transcripts, are preferentially expressed in oocytes (Vallee et al. 2006). Furthermore, an important molecular characteristic of germ cells was also reported: germ cell-specific regulation of core promoter-associated transcription factors is conserved between Xenopus and mice (Xiao et al. 2006). Tbp/2/Trf3 and Gtf2a1/f/Alf are demonstrated to be expressed preferentially in oocytes and can form in vitro core promoter complexes with TBP and TFIIA. Therefore, identifying other germ cell-specific transcription factors is necessary to understand the genetic cascades that drive oocyte development and folliculogenesis.

\section{Comparison of oocytes with ES cells in terms of their gene expression profiles}

Recent studies on cell fusion between a somatic cell and an ES cell suggest that cytoplasm of ES cells can reprogram an introduced somatic nucleus to confer pluripotency. In this aspect, the cytoplasmic environments of ES cells and oocytes share the capacity to reprogram a somatic nucleus (Tada et al. 2001, Cowan et al. 2005). Accordingly, a set of genes commonly expressed in oocytes and ES cells are likely responsible for reprograming somatic cells. To identify these genes, gene expression profiling data of human oocytes and human ES cells were explored (Kocabas et al. 2006, Zhang et al. 2007). Compared with reference samples, 5331 and 1626 transcripts are significantly upregulated in human oocytes and ES cells respectively (Kocabas et al. 2006). When the genes differentially upregulated in human ES cells are intersected with those differentially upregulated in human oocytes, 388 transcripts are overlapped. This list of genes, including POU5F1/OCT4, DNMT3b, DAZL, and high-mobility group proteins (HMGB2, HMGB3, and HMGN4) (Kocabas et al. 2006), may provide good candidate genes for the future studies on molecular mechanisms of nuclear reprograming.

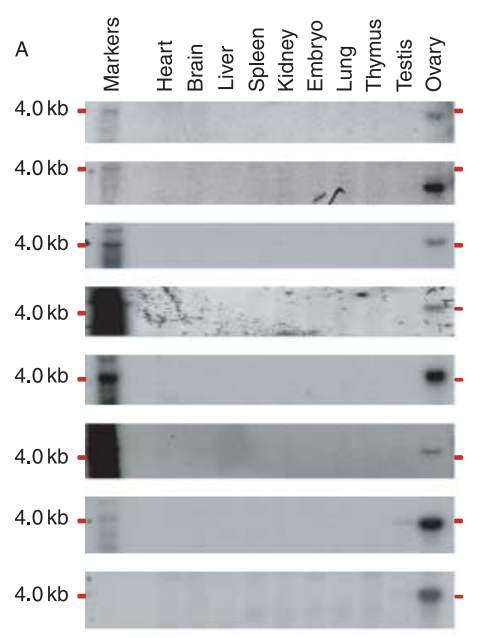

Nalp- $\alpha$
(NIrp4c)
Nalp- $\gamma$
(Nlrp4b)
Nalp-
(NIrp9b)
Nalp- $\zeta$
(NIrp9c)
Nalp- $\eta$
(NIrp4a)
Nalp- $\theta$
(NIrp9a)
Nalp-1
(Nlrp14)
Nalp-k
(NIrp4f)

Reproduction (2008) 135 581-592

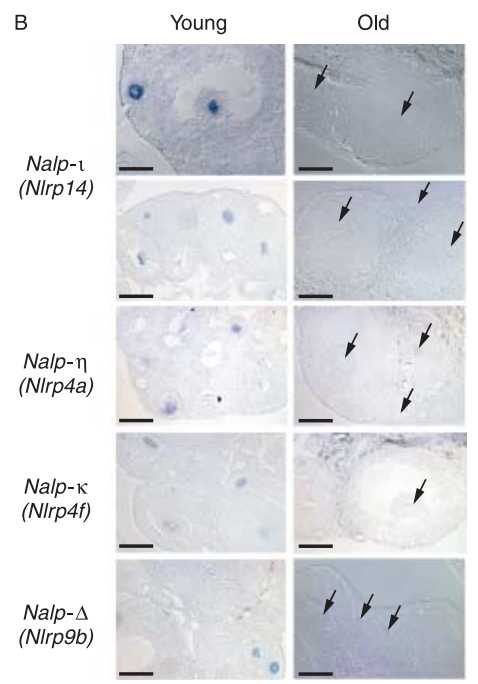

Figure 6 Oocyte-specific expression of the novel Nalp-family mouse genes (reprinted from 'Ageassociated alteration of gene expression patterns in mouse oocytes', Hamatani T et al. 2004 Human Molecular Genetics 13 2263-2278, with permission from Oxford University Press). (A) Northern blot analysis shows their ovary-specific expression and (B) in situ hybridization shows their oocytespecific expression on ovary sections. 
On the other hand, 'induced pluripotent stem cells (iPS cells)' were recently generated by forced expression of defined factors: Pou5f1/Oct4, Sox2, Klf4, and Myc (Takahashi \& Yamanaka 2006). Surprisingly, iPS cells selected by Nanog expression are capable of germ cell transmission (Okita et al. 2007). These iPS factors, however, show little maternal expression in oocytes (except in the case of Oct4) and increased zygotic expression during preimplantation stages (except in the case of $\mathrm{Myc}$ ), based on EST frequencies in Unigene cDNA libraries and microarray data during oogenesis to preimplantation development (Fig. 7). Therefore, the mechanism of oocytes to induce pluripotency is likely different from that of ES cells. Although the genes commonly expressed in oocytes and ES cells are not necessarily important to induce pluripotency, maternal factors that can induce zygotic expression of the 'iPS factors' (Oct4, Sox2, and Klf4) are rather more substantial in oocytes.

\section{Perspective}

Oocytes offer a relatively homogeneous biological system that is well adapted to gene expression profiling studies: arrest of cell cycle at the metaphase II stage, quiescence in transcription after germinal vesicle breakdown, and little contamination in oocyte samples with any other types of cells after thorough removal of cumulus cells. There are, however, several limitations in applying microarray technologies to study the molecular mechanisms in oocytes and preimplantation embryos. Although the recent advent of linear RNA amplification (in vitro transcription-based protocols) and exponential amplification (PCR-based strategies) techniques allowed several groups to study oocyte transcriptomes using a tiny amount of RNA even in an individual oocyte (Bermudez et al. 2004, Dobson et al. 2004, Li et al. 2006, Jones et al. 2007), the efficacy of RNA amplification is not yet good enough to analyze an individual blastomere of preimplantation embryos. Furthermore, poly(A) length affects efficiency of RNA amplification. Although the synthesis of new transcripts essentially ceases after germinal vesicle breakdown, poly $(A)$ tails of some classes of existing transcripts in oocytes are elongated, leading to increased translation and protein levels (Bachvarova 1992). Thus, regulation of the poly(A) tail length is a major mechanism for controlling maternal transcript activity. Unlike the T7-oligo(dT) primers used in the conventional linear RNA amplification procedures, the uniquely designed Full Spectrum MultiStart Primers for in vitro transcription from System Biosciences (Mountain View, CA, USA) initiates cDNA synthesis at multiple points along mRNAs with little or no bias with respect to the length of poly(A) tails. Transcript profiles generated from microarray studies using this modified RNA amplification protocol would provide a more accurate perspective of the
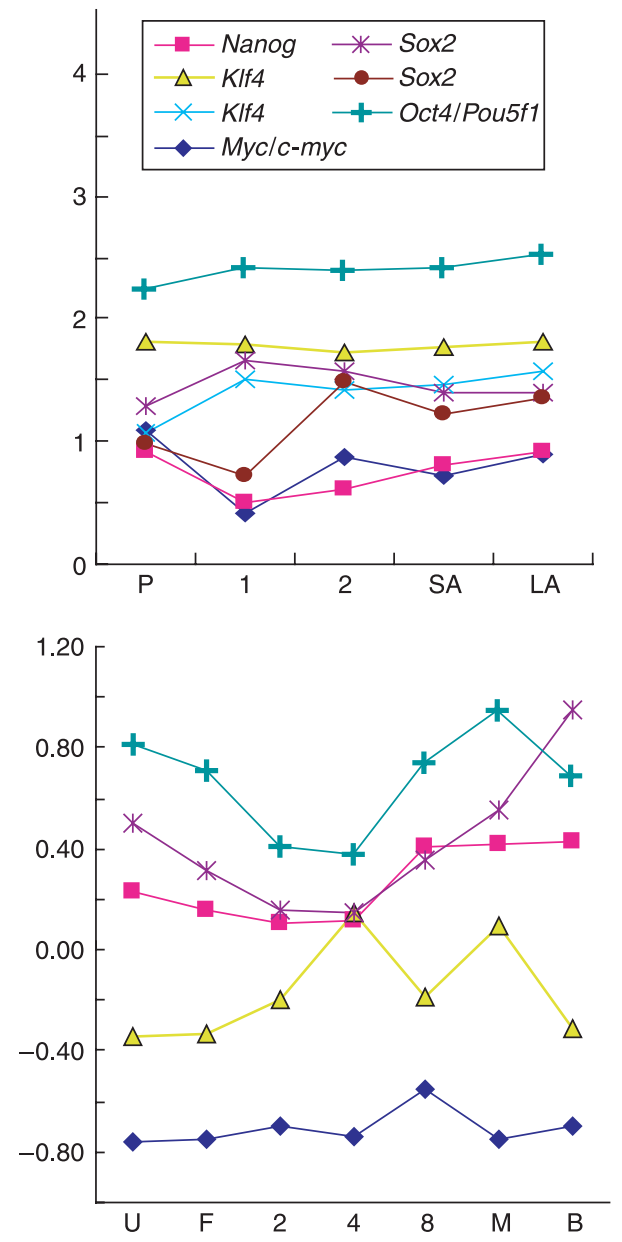

Figure 7 The gene expression changes of the iPS factors based on the published microarray data. P, 1, 2, SA, and LA represent the primordial follicle stage, the primary follicle stage, the secondary follicle stage, the small antral follicle stage, and the large antral follicle stage respectively. $U, F, 2,4,8, M$, and B denote unfertilized egg, fertilized egg, two-cell embryo, four-cell embryo, eight-cell embryo, morula, and blastocyst respectively.

global changes in populations of both degrading and stable transcripts during oocyte maturation and ZGA (Su et al. 2007).

'Tailor-made regenerative medicine' includes nuclear transfer from a patient's somatic cell to an enucleated donated oocyte, development of the reconstructed embryo up to the blastocyst stage, and establishment of the patient's ES cells. 'Making oocytes' will be an essential technique to develop 'tailor-made regenerative medicine' that needs large quantities of healthy ooplasms. 'Oocyte-like' cells were recently grown and isolated by utilizing GFP expression as a selection marker during differentiation of ES cells containing GFP expression cassettes under the Pou5f1 promoter (Hubner et al. 2003). Nobody, however, has succeeded in generating oocytes by manipulating gene expression in ES and somatic cells. Even though forced expression of a set of several transcription factors in ES cells 
may allow us to generate an oocyte, there is a problem in the oocyte; its nucleus ought to have genetic abnormalities. In contrast, its ooplasm might contain all the gene products that can support embryonic development after fertilization. Unlike another strategy using iPS cells that cannot avoid transmitting genetic abnormalities, the ooplasm can be safely used for 'tailor-made regenerative medicine' or 'ooplasmic donation'.

Since transcriptional cascades that activate an oocyte-specific developmental program are largely unknown, a set of master genes that drive the cascades have not yet been defined. Oocyte-specific transcription factors, however, are likely to be the critical switches for the differentiation into oocytes and good candidates for manipulation of gene expression. For example, NOBOX binds to the NOBOX binding elements with high affinity and augments transcriptional activity of mouse Pou5f1 and Gdf9 promoters (Choi \& Rajkovic 2006). Other examples are factor in germ cell (FIGLA) and $\mathrm{SOHLH} 1$ that bind to E-box. They are suggested to increase transcriptional activity of Zp1-3, which have

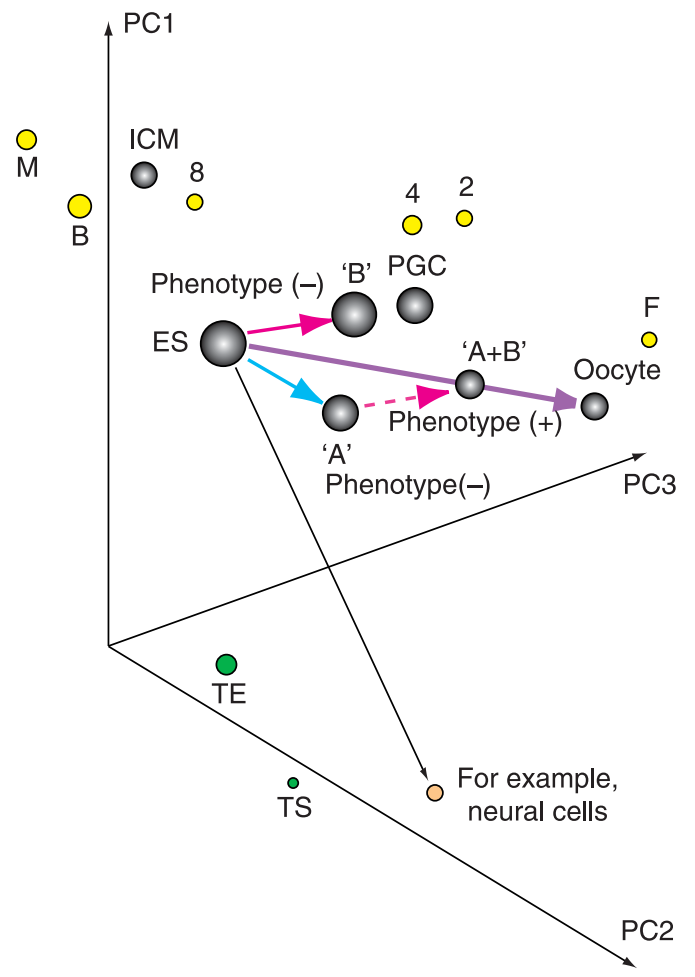

Figure 8 Explanation of a model to transform ES cells efficiently into oocytes using gene expression profiling as a guide. PC, principle component; PGC, primordial germ cell; ICM, inner cell mass; TE, trophectoderm; F, fertilized egg; 2, two-cell embryo; 4, four-cell embryo; 8, eight-cell embryo; $M$, morula; $B$, blastocyst. If over-expression of 'gene $\mathrm{A}^{\prime}$ makes a global expression profile of ES cells closer to that of oocytes in the PCA coordinate, 'gene A' could be a good candidate to promote the oocyte developmental program. Even though no changes in phenotypes of ES cells are observed with over-expression of 'gene $\mathrm{A}^{\text {', }}$ forced expression of 'gene $A$ ' plus that of 'gene $B$ ' in ES cells may show a distinctive phenotype including oocytes or follicles. promoters including E-box (Yan et al. 2006: Pangas, 2006 \#613).

On the other hand, nobody pays attention to a transcription factor whose knockout showed no distinctive phenotypes. Nonetheless, recent advent in microarray technologies allows us to catch any changes in a gene expression profile of cells transfected with a construct to modify gene expression. If a gene expression profile of ES cells approaches that of oocytes in the PCA coordinate, in spite of no phenotypic change, by upregulation of a certain transcription factor, the transcription factor is likely a candidate gene as a tool to induce the oocyte developmental program (Fig. 8). Further forced expression of another transcription factor in the ES cell may result in a similar gene expression profile to that of oocytes and then may achieve a certain remarkable phenotype including follicles or oocytes. Such synergy between cell biology and bioinformatics will become more important and beneficial to establish an in vitro oocyte-development model to 'make an oocyte'.

\section{Acknowledgements}

The authors declare that there is no conflict of interest that would prejudice the impartiality of this scientific work.

\section{References}

Abe H 2007 A non-invasive and sensitive method for measuring cellular respiration with a scanning electrochemical microscopy to evaluate embryo quality. Journal of Mammalian Ova Research 24 70-78.

Adjaye J 2005 Whole-genome approaches for large-scale gene identification and expression analysis in mammalian preimplantation embryos. Reproduction, Fertility, and Development 17 37-45.

Armstrong DT 2001 Effects of maternal age on oocyte developmental competence. Theriogenology 55 1303-1322.

Ashburner M, Ball CA, Blake JA, Botstein D, Butler H, Cherry JM, Davis AP, Dolinski K, Dwight SS, Eppig JT, et al. 2000 Gene ontology: tool for the unification of biology. The Gene Ontology Consortium. Nature Genetics 25 25-29.

ASRM/SART 2000 Assisted reproductive technology in the United States: 1997 results generated from the American Society for Reproductive Medicine/Society for Assisted Reproductive Technology Registry. Fertility and Sterility 74 641-653 (discussion 653-644).

Assou S, Anahory T, Pantesco V, Le Carrour T, Pellestor F, Klein B, Reyftmann L, Dechaud H, De Vos J \& Hamamah S 2006 The human cumulus-oocyte complex gene-expression profile. Human Reproduction 21 1705-1719.

Bachvarova RF 1992 A maternal tail of poly(A): the long and the short of it. Cell 69 895-897.

Bermudez MG, Wells D, Malter H, Munne S, Cohen J \& Steuerwald NM 2004 Expression profiles of individual human oocytes using microarray technology. Reproductive Biomedicine Online 8 325-337.

Burns KH, Viveiros MM, Ren Y, Wang P, DeMayo FJ, Frail DE, Eppig JJ \& Matzuk MM 2003 Roles of NPM2 in chromatin and nucleolar organization in oocytes and embryos. Science 300 633-636.

Carter MG, Hamatani T, Sharov AA, Carmack CE, Qian Y, Aiba K, Ko NT, Dudekula DB, Brzoska PM, Hwang SS, et al. 2003 In situ-synthesized novel microarray optimized for mouse stem cell and early developmental expression profiling. Genome Research 13 1011-1021. 
Choi Y \& Rajkovic A 2006 Characterization of NOBOX DNA binding specificity and its regulation of Gdf9 and Pou5f1 promoters. Journal of Biological Chemistry 281 35747-35756.

Cohen J, Scott R, Schimmel T, Levron J \& Willadsen S 1997 Birth of infant after transfer of anucleate donor oocyte cytoplasm into recipient eggs. Lancet 350 186-187.

Cohen J, Scott R, Alikani M, Schimmel T, Munne S, Levron J, Wu L, Brenner C, Warner C \& Willadsen S 1998 Ooplasmic transfer in mature human oocytes. Molecular Human Reproduction 4 269-280.

Cowan CA, Atienza J, Melton DA \& Eggan K 2005 Nuclear reprogramming of somatic cells after fusion with human embryonic stem cells. Science 309 1369-1373.

Cui XS, Li XY, Yin XJ, Kong IK, Kang JJ \& Kim NH 2007 Maternal gene transcription in mouse oocytes: genes implicated in oocyte maturation and fertilization. Journal of Reproduction and Development 53 405-418.

Dade S, Callebaut I, Mermillod P \& Monget P 2003 Identification of a new expanding family of genes characterized by atypical LRR domains. Localization of a cluster preferentially expressed in oocyte. FEBS Letters 555 533-538.

Dahlquist KD, Salomonis N, Vranizan K, Lawlor SC \& Conklin BR 2002 GenMAPP, a new tool for viewing and analyzing microarray data on biological pathways. Nature Genetics 31 19-20.

Dean J 2002 Oocyte-specific genes regulate follicle formation, fertility and early mouse development. Journal of Reproductive Immunology 53 171-180.

Dessie SW, Rings F, Holker M, Gilles M, Jennen D, Tholen E, Havlicek V, Besenfelder U, Sukhorukov VL, Zimmermann U, et al. 2007 Dielectrophoretic behavior of in vitro-derived bovine metaphase II oocytes and zygotes and its relation to in vitro embryonic developmental competence and mRNA expression pattern. Reproduction 133 931-946.

Dobson AT, Raja R, Abeyta MJ, Taylor T, Shen S, Haqq C \& Pera RA 2004 The unique transcriptome through day 3 of human preimplantation development. Human Molecular Genetics 13 1461-1470.

Dong J, Albertini DF, Nishimori K, Kumar TR, Lu N \& Matzuk MM 1996 Growth differentiation factor-9 is required during early ovarian folliculogenesis. Nature 383 531-535.

Doniger SW, Salomonis N, Dahlquist KD, Vranizan K, Lawlor SC \& Conklin BR 2003 MAPPFinder: using gene ontology and GenMAPP to create a global gene-expression profile from microarray data. Genome Biology 4 R7.

Engmann L, Maconochie N, Sladkevicius P, Bekir J, Campbell S \& Tan SL 1999 The outcome of in vitro fertilization treatment in women with sonographic evidence of polycystic ovarian morphology. Human Reproduction 14 167-171.

Evsikov AV, Graber JH, Brockman JM, Hampl A, Holbrook AE, Singh P, Eppig JJ, Solter D \& Knowles BB 2006 Cracking the egg: molecular dynamics and evolutionary aspects of the transition from the fully grown oocyte to embryo. Genes and Development 20 2713-2727.

Fragouli E, Wells D, Thornhill A, Serhal P, Faed MJ, Harper JC \& Delhanty JD 2006 Comparative genomic hybridization analysis of human oocytes and polar bodies. Human Reproduction 21 2319-2328.

Galloway SM, McNatty KP, Cambridge LM, Laitinen MP, Juengel JL, Jokiranta TS, McLaren RJ, Luiro K, Dodds KG, Montgomery GW, et al. 2000 Mutations in an oocyte-derived growth factor gene (BMP15) cause increased ovulation rate and infertility in a dosage-sensitive manner. Nature Genetics 25 279-283.

Gasca S, Pellestor F, Assou S, Loup V, Anahory T, Dechaud H, De Vos J \& Hamamah S 2007 Identifying new human oocyte marker genes: a microarray approach. Reproductive Biomedicine Online 14 175-183.

Hamatani T, Carter MG, Sharov AA \& Ko MS 2004a Dynamics of global gene expression changes during mouse preimplantation development. Developmental Cell 6 117-131.

Hamatani T, Falco G, Carter MG, Akutsu H, Stagg CA, Sharov AA, Dudekula DB, VanBuren V \& Ko MS 2004b Age-associated alteration of gene expression patterns in mouse oocytes. Human Molecular Genetics $132263-2278$.

Hennebold JD, Tanaka M, Saito J, Hanson BR \& Adashi EY 2000 Ovaryselective genes I: the generation and characterization of an ovaryselective complementary deoxyribonucleic acid library. Endocrinology 141 2725-2734.
Hodgman R, Tay J, Mendez R \& Richter JD 2001 CPEB phosphorylation and cytoplasmic polyadenylation are catalyzed by the kinase IAK1/Eg2 in maturing mouse oocytes. Development 128 2815-2822.

Hubner K, Fuhrmann G, Christenson LK, Kehler J, Reinbold R, De La Fuente R, Wood J, Strauss JF III, Boiani M \& Scholer HR 2003 Derivation of oocytes from mouse embryonic stem cells. Science 300 1251-1256.

Hughes TR, Mao M, Jones AR, Burchard J, Marton MJ, Shannon KW, Lefkowitz SM, Ziman M, Schelter JM, Meyer MR, et al. 2001 Expression profiling using microarrays fabricated by an ink-jet oligonucleotide synthesizer. Nature Biotechnology 19 342-347.

Johnson MH, Lim A, Fernando D \& Day ML 2002 Circadian clockwork genes are expressed in the reproductive tract and conceptus of the early pregnant mouse. Reproductive Biomedicine Online 4 140-145.

Jones GM, Song B, Cram DS \& Trounson AO 2007 Optimization of a microarray based approach for deriving representative gene expression profiles from human oocytes. Molecular Reproduction and Development 74 8-17.

Klein J \& Sauer MV 2001 Assessing fertility in women of advanced reproductive age. American Journal of Obstetrics and Gynecology $185758-770$.

Ko MS 2004 Embryogenomics of pre-implantation mammalian development: current status. Reproduction, Fertility, and Development 16 79-85.

Ko MS, Kitchen JR, Wang X, Threat TA, Hasegawa A, Sun T, Grahovac MJ, Kargul GJ, Lim MK, Cui Y, et al. 2000 Large-scale cDNA analysis reveals phased gene expression patterns during preimplantation mouse development. Development 127 1737-1749.

Kocabas AM, Crosby J, Ross PJ, Otu HH, Beyhan Z, Can H, Tam WL, Rosa GJ, Halgren RG, Lim B, et al. 2006 The transcriptome of human oocytes. PNAS 103 14027-14032.

van Kooij RJ, Looman CW, Habbema JD, Dorland M \& te Velde E 1996 Age-dependent decrease in embryo implantation rate after in vitro fertilization. Fertility and Sterility 66 769-775.

Li SS, Liu YH, Tseng CN \& Singh S 2006 Analysis of gene expression in single human oocytes and preimplantation embryos. Biochemical and Biophysical Research Communications 340 48-53.

Lopatina N, Haskell JF, Andrews LG, Poole JC, Saldanha S \& Tollefsbol T 2002 Differential maintenance and de novo methylating activity by three DNA methyltransferases in aging and immortalized fibroblasts. Journal of Cellular Biochemistry 84 324-334.

Ludwig M, Finas DF, al-Hasani S, Diedrich K \& Ortmann O 1999 Oocyte quality and treatment outcome in intracytoplasmic sperm injection cycles of polycystic ovarian syndrome patients. Human Reproduction 14 354-358.

Mager J, Schultz RM, Brunk BP \& Bartolomei MS 2006 Identification of candidate maternal-effect genes through comparison of multiple microarray data sets. Mammalian Genome 17 941-949.

Mendez R \& Richter JD 2001 Translational control by CPEB: a means to the end. Nature Reviews. Molecular Cell Biology 2 521-529.

Mulders AG, Laven JS, Imani B, Eijkemans MJ \& Fauser BC 2003 IVF outcome in anovulatory infertility (WHO group 2) - including polycystic ovary syndrome - following previous unsuccessful ovulation induction. Reproductive Biomedicine Online 7 50-58.

Navot D, Bergh PA, Williams MA, Garrisi GJ, Guzman I, Sandler B \& Grunfeld L 1991 Poor oocyte quality rather than implantation failure as a cause of age-related decline in female fertility. Lancet 337 1375-1377.

Nothias JY, Majumder S, Kaneko KJ \& DePamphilis ML 1995 Regulation of gene expression at the beginning of mammalian development. Journal of Biological Chemistry $27022077-22080$.

Okita K, Ichisaka T \& Yamanaka S 2007 Generation of germline-competent induced pluripotent stem cells. Nature 448 313-317.

Paillisson A, Dade S, Callebaut I, Bontoux M, Dalbies-Tran R, Vaiman D \& Monget P 2005 Identification, characterization and metagenome analysis of oocyte-specific genes organized in clusters in the mouse genome. $B M C$ Genomics 676.

Pan H, O'Brien MJ, Wigglesworth K, Eppig JJ \& Schultz RM 2005 Transcript profiling during mouse oocyte development and the effect of gonadotropin priming and development in vitro. Developmental Biology 286 493-506.

Patrizio P, Fragouli E, Bianchi V, Borini A \& Wells D 2007 Molecular methods for selection of the ideal oocyte. Reproductive Biomedicine Online 15 346-353. 
Rajkovic A, Yan MSC, Klysik M \& Matzuk M 2001 Discovery of germ cellspecific transcripts by expressed sequence tag database analysis. Fertility and Sterility 76 550-554.

Schultz RM 2002 The molecular foundations of the maternal to zygotic transition in the preimplantation embryo. Human Reproduction Update 8 323-331.

Sharov AA, Piao Y, Matoba R, Dudekula DB, Qian Y, VanBuren V, Falco G, Martin PR, Stagg CA, Bassey UC, et al. 2003 Transcriptome analysis of mouse stem cells and early embryos. PLoS Biology 1 E74.

Steuerwald NM, Bermudez MG, Wells D, Munne S \& Cohen J 2007 Maternal age-related differential global expression profiles observed in human oocytes. Reproductive Biomedicine Online 14 700-708.

Su YQ, Sugiura K, Woo Y, Wigglesworth K, Kamdar S, Affourtit J \& Eppig JJ 2007 Selective degradation of transcripts during meiotic maturation of mouse oocytes. Developmental Biology 302 104-117.

Suzumori N, Burns KH, Yan W \& Matzuk MM 2003 RFPL4 interacts with oocyte proteins of the ubiquitin-proteasome degradation pathway. PNAS 100 550-555.

Tada M, Takahama Y, Abe K, Nakatsuji N \& Tada T 2001 Nuclear reprogramming of somatic cells by in vitro hybridization with ES cells. Current Biology 11 1553-1558.

Takahashi K \& Yamanaka S 2006 Induction of pluripotent stem cells from mouse embryonic and adult fibroblast cultures by defined factors. Cell $126663-676$.

Takeuchi T, Ergun B, Huang TH, Rosenwaks Z \& Palermo GD 1999 A reliable technique of nuclear transplantation for immature mammalian oocytes. Human Reproduction 14 1312-1317.

Terada Y, Simerly C \& Schatten G 2000 Microfilament stabilization by jasplakinolide arrests oocyte maturation, cortical granule exocytosis, sperm incorporation cone resorption, and cell-cycle progression, but not DNA replication, during fertilization in mice. Molecular Reproduction and Development $\mathbf{5 6}$ 89-98.

Tong ZB, Gold L, Pfeifer KE, Dorward H, Lee E, Bondy CA, Dean J \& Nelson LM 2000 Mater, a maternal effect gene required for early embryonic development in mice. Nature Genetics 26 267-268.

Vallee M, Robert C, Methot S, Palin MF \& Sirard MA 2006 Cross-species hybridizations on a multi-species cDNA microarray to identify evolutionarily conserved genes expressed in oocytes. BMC Genomics 7113.

Wang QT, Piotrowska K, Ciemerych MA, Milenkovic L, Scott MP, Davis RW \& Zernicka-Goetz M 2004 A genome-wide study of gene activity reveals developmental signaling pathways in the preimplantation mouse embryo. Developmental Cell 6 133-144.
Wells D, Escudero T, Levy B, Hirschhorn K, Delhanty JD \& Munne S 2002 First clinical application of comparative genomic hybridization and polar body testing for preimplantation genetic diagnosis of aneuploidy. Fertility and Sterility 78 543-549.

Wood JR, Dumesic DA, Abbott DH \& Strauss JF III 2007 Molecular abnormalities in oocytes from women with polycystic ovary syndrome revealed by microarray analysis. Journal of Clinical Endocrinology and Metabolism 92 705-713.

Wu X, Viveiros MM, Eppig J, Bai Y, Fitzpatrick SL \& Matzuk MM 2003 Zygote arrest 1 (Zar1) is a novel maternal-effect gene critical for the oocyte-to-embryo transition. Nature Genetics 33 187-191.

Xiao L, Kim M \& DeJong J 2006 Developmental and cell type-specific regulation of core promoter transcription factors in germ cells of frogs and mice. Gene Expression Patterns 6 409-419.

Yan C, Elvin JA, Lin YN, Hadsell LA, Wang J, DeMayo FJ \& Matzuk MM 2006 Regulation of growth differentiation factor 9 expression in oocytes in vivo: a key role of the E-box. Biology of Reproduction 74 999-1006.

Yoon SJ, Kim KH, Chung HM, Choi DH, Lee WS, Cha KY \& Lee KA 2006 Gene expression profiling of early follicular development in primordial, primary, and secondary follicles. Fertility and Sterility 85 193-203.

Zeng F \& Schultz RM 2003 Gene expression in mouse oocytes and preimplantation embryos: use of suppression subtractive hybridization to identify oocyte- and embryo-specific genes. Biology of Reproduction $6831-39$.

Zeng F, Baldwin DA \& Schultz RM 2004 Transcript profiling during preimplantation mouse development. Developmental Biology 272 483-496.

Zhang P, Kerkela E, Skottman H, Levkov L, Kivinen K, Lahesmaa R, Hovatta O \& Kere J 2007 Distinct sets of developmentally regulated genes that are expressed by human oocytes and human embryonic stem cells. Fertility and Sterility 87 677-690.

Received 22 September 2007

First decision 31 October 2007

Revised manuscript received 1 February 2008

Accepted 27 February 2008 